\title{
Philosophiques
}

\section{Le théorème de Gödel. Traductions de l'anglais et de l'allemand par Jean-Baptiste Scherrer. Sources du savoir, Paris : Éditions du Seuil, 1989, 170 p.}

\section{Yvon Gauthier}

Volume 17, numéro 1, printemps 1990

URI : https://id.erudit.org/iderudit/027112ar

DOI : https://doi.org/10.7202/027112ar

Aller au sommaire du numéro

Éditeur(s)

Société de philosophie du Québec

ISSN

0316-2923 (imprimé)

1492-1391 (numérique)

Découvrir la revue

Citer ce compte rendu

Gauthier, Y. (1990). Compte rendu de [Le théorème de Gödel. Traductions de

l'anglais et de l'allemand par Jean-Baptiste Scherrer. Sources du savoir, Paris :

Éditions du Seuil, 1989, 170 p.] Philosophiques, 17(1), 157-159.

https://doi.org/10.7202/027112ar d'utilisation que vous pouvez consulter en ligne.

https://apropos.erudit.org/fr/usagers/politique-dutilisation/ 
Le théorème de Gödel. Traductions de l'anglais et de l'allemand par JeanBaptiste Scherrer. Sources du savoir, Paris : Éditions du Seuil, 1989, 170 p.

$$
\text { par Yvon Gauthier }
$$

Ce recueil contient la première traduction française du texte du théorème d'incomplétude que Gödel a publié originellement sous le titre « Über formal unentscheidbare Sätze der Principia Mathematica und verwandter Systeme I " dans les Monatshefte für Mathematik und Physik 38, 173-198.

Il contient aussi la traduction du texte de Ernest Nagel et James R. Newman sur la preuve de Gödel, Gödel's Proof (New York : New York University Press, 1958).

Des commentaires de Jean-Yves Girard sur « Le champ du signe ou la faillite du réductionnisme » complètent l'ouvrage. De la traduction du texte de Gödel, il y a peu à dire, sinon qu'elle sera peu utile. Les logiciens ont accès depuis longtemps à des traductions en anglais et la traduction sans commentaire critique, contrairement par exemple aux Collected Works, vol. I, (édité par Oxford University Press : New York, 1986 sous la direction de S. Feferman), ne saurait avoir l'intérêt souhairé. Remarquons cependant que le travail est soigné, même s'il adopte la traduction «satisfiable», pour l'allemand " erfüllbar »; on devrait traduire l'anglais " satisfiable " par « satisfaisable", même si le terme correct est plus lourd. 
La traduction de l'ouvrage de Nagel et Newman semble justifiée par le caractère vulgarisateur de l'ouvrage; mais une présentation plus ou moins formelle et renouvelée eût sans doute été plus riche d'enseignement que le texte déjà anachronique de nos deux auteurs. Jean-Yves Girard ne manque pas d'ailleurs de relever l'incongruité de leur présence dans ce recueil. Mais il y va lui-même de remarques excessives et de jugements simplificateurs qu'il n'est peut-être pas vain de relever rapidement.

Il s'agit essentiellement du programme de Hilbert : donner une preuve finitaire de la consistance de l'analyse classique en se fondant uniquement sur des objets combinatoires, les expressions formelles. Finitisme et formalisme entraînent le réductionnisme que combat Gödel. Or s'il est vrai que les deux théorèmes d'incomplétude, le second portant sur les preuves de consistance, réduisent considérablement l'ambitieux projet de Hilbert, ils ne le ruinent pas. Kreisel, en particulier, a insisté sur un programme de Hilbert révisé. C'est d'ailleurs dans cette direction que vont les importants travaux d'Edward Nelson en arithmétique prédicative (E. Nelson Predicative Arithmetic, Princeton: N. J., Princeton University Press, 1986). Avec l'arithmétique prédicative, on peut démontrer l'auto-consistance de l'arithmétique de $R$. Robinson, ce qui nous permet de fonder syntaxiquement l'analyse non standard. Évidemment, c'est l'arithmétique de Peano qui est incomplète au sens de Gödel, justement parce que le postulat d'induction n'est pas prédicatif. Prédicatif signifie essentiellement induction bornée ou quantification bornée, comme le note Girard (p. 152) dans sa critique du programme de Hilbert. Or la logique dynamique ou non monotone qu'il promeut (pp. 170-171) comme les fonctions élémentairement calculables de l'informatique théorique qu'il défend exigent des bornes logarithmiques puisque l'exponentiation ne peut $y$ être totale. Le théorème de Gödel n'est assurément pas de nature prédicative, même s'il contient une bonne mesure de constructivité. La démonstration du théorème VII, par exemple, qui énonce que «Toute relation récursive est arithmétique » que l'on trouve en page 133 du présent recueil fait appel au théorème du reste chinois et dans la note de la page 134, f est une variable définie sur tous les nombre naturels (elle n'est pas bornée). D'autres détails - la notion de degré, de diagonalisation - jettent un peu plus d'ombre sur le caractère constructif de la preuve.

Quoi qu'il en soit, du point de vue historique, Hilbert a été la source et l'inspiration de Gödel. Non seulement les Grundzüge der theoretischen Logik de Hilbert et Ackermann, mais les cours de Carnap, néo-positiviste s'il en est, ont eu la plus grande influence. D'un point de vue historique, encore, lorsque Girard pourfend les chercheurs de nouveaux axiomes à la suite du théorème de Gödel, il oublie de dire que c'est Gödel lui-même qui a ouvert la piste, en ce qui touche en particulier les axiomes forts d'infinité de la théorie des ensembles. Il est vrai que les résultats importants de l'histoire de la logique sont des résultats négatifs, incomplétude, indécidabilité, indépendance, mais il est excessif de penser que la plus grande contribution positive en logique est le Hauptsatz de Gentzen sur l'élimination des coupures. Girard, 
lui-même un logicien fort respectable et spécialiste de la théorie des démonstrations, oublie un peu vite toute la théorie des modèles... Faur-il rappeler que A. Robinson était formaliste et que Gödel pensait le plus grand bien de ses travaux.

Je termine sur ces notes anecdotiques pour montrer les limites de la popularisation. En traduisant pour le public français le texte de Gödel, on a voulu le rendre accessible; on y a accolé Nagel et Newman et Jean-Yves Girard qui se sent un peu mal à l'aise dans ce rôle de médiateur, sinon de communicateur. C'est une réussite mitigée.

Département de philosophie

Université de Montréal 